\title{
Europe plans molecular screening center for translational research
}

Almost a decade ago, the US National Institutes of Health kicked off its Molecular Libraries Initiative to provide academic researchers with access to the high-throughput screening tools needed to identify new therapeutic compounds. Europe now seems keen on catching up.

Last month, the Innovative Medicines Initiative (IMI), a $€ 2$ billion ( $\$ 2.6$ billion) Brussels-based partnership between the European Commission and the European Federation of Pharmaceutical Industries and Associations (EFPIA), invited proposals to build a molecular screening facility for drug discovery in Europe that will combine the inquisitiveness of academic scientists with industry know-how. The IMI's call for tenders says the facility will counter "fragmentation" between these sectors.

The IMI will offer $€ 80$ million over five years to the consortium selected to build up and manage a centralized screening infrastructure and a library of up to 500,000 compounds. Expressions of interest from qualified academic-industry partnerships are due in mid-May.

Major pharmaceutical companies have more experience with high-throughput screening than do most academic institutes. Yet companies often limit tests of their closely held candidate chemicals to a fraction of potential disease targets. By pooling chemical libraries and screening against a more diverse set of targets-and identifying more molecular interactions-both academics and pharmaceutical companies stand to gain, says Hugh Laverty, an IMI project manager.

The IMI already has commitments from seven members of the EFPIA to contribute 50,000 compounds each, and future academic partners are expected to donate between 100,000 and 200,000 total. (The NIH similarly has around 300,000 chemically diverse compounds in its small-molecule repository.)

Onlookers note the challenge of handling so many molecular candidates. Whoever manages the IMI's 'European Screening Centre' will need to develop an efficient process for preselecting compounds to make the high-throughput screening worth the cost to taxpayers, says Robert Damoiseaux, scientific director of the Molecular Screening Shared Resource (MSSR) at the University of California-Los Angeles. Damoiseaux recommends that Europeans look across the pond for insight on this: "It's certainly really useful to compare notes with American researchers and have a look at which lessons

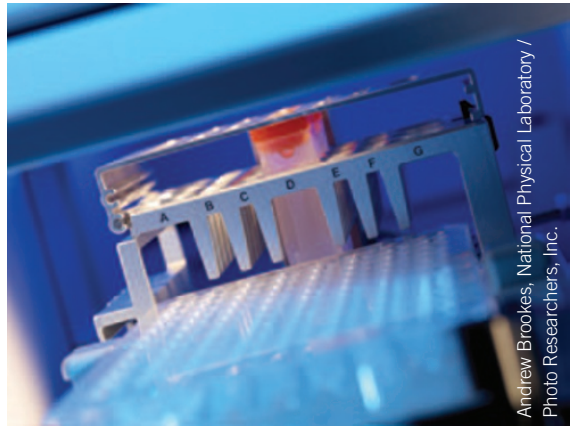

Need for speed: High-throughput discovery.

can be learned, what paradigms worked and what didn't."

Ultimately, a facility as large as the proposed
European Screening Center should be more efficient than the smaller sites that already exist around Europe, says Gerardo Turcatti, who directs the Biomolecular Screening Facility at the Swiss Federal Institute of Technology in Lausanne. He adds, however, that sorting out intellectual property in any collaboration that also involves industrial applications will be a challenge.

Laverty says the IMI will help the winning consortium negotiate the particulars with the industry partners. "We're very aware of intellectual property concerns," he explains, adding that identifying a promising compound might trigger milestone payments to a compound's contributor.

Lucas Laursen

\section{Analysis of drug failures underscores value of robust phase 2 testing}

In the realm of drug development, many pharmaceutical companies-and their shareholders - are especially eager to reach phase 3 clinical trials, where candidate drugs are tested for their ultimate effectiveness. But an analysis presented last month at a meeting of the New Jersey chapter of the Licensing Executive Society emphasizes that companies are wiser to spend more resources working on finetuning their therapy in phase 2 , lest they falter just in sight of the finish line.

"It's not sexy to hang out in phase 2 for three to four years, but that may be best way to develop a drug," says Lisa Natanson, an analyst at Deloitte Recap, the San Francisco area-based, biopharma-focused intelligence arm of the international consulting firm Deloitte Touche Tohmatsu.

Last year, Natanson and her colleagues combed through information in the Deloitte Recap database from 190 mostly US-based biomedical companies on 97 compounds originated in house that reached a final clinical outcome in the US within the past decade. The analysis revealed that the 64 approved drugs enrolled a median of 171 participants in each phase 2 program and conducted two phase 2 trials, on average. By comparison, the late-stage failures-which were terminated at the phase 3 stage or rejected by the US Food and Drug Administration for problems with efficacy or safety reasons-enrolled a median of 69 people per phase 2 program and conducted an average of only 1.2 such trials, according to the Deloitte report. Moreover, smaller phase 2 trials did not reduce the time it took to help companies reach a final result.

Steven Paul, who retired in 2009 as president of the Lilly Research Laboratories in Indianapolis, says that the Deloitte data are consistent with his experience. He explains that business attitudes focused on near-term results are partly to blame. A primary reason for premature advancement to phase 3 , he says, is "wishful thinking."

But rolling out larger phase 2 studies is easier said than done for cash-strapped startups. To that effect, John Arrowsmith, an advisor at Thomson Reuters Life Sciences Consulting in New York, suggests that small companies that might lack funding for large phase 2 trials can conduct a preliminary version to get an early indication of efficacy as a way to attract investor interest. "It isn't a case of all or nothing in phase 2. You can get a sniff of success by running an investigative trial rather than a full-blown phase 2 study," Arrowsmith says.

Roxanne Palmer 